\title{
Soft Metal Gains Hulk-Like Strength
}

\author{
When rapidly compressed to planetary-core pressures, lead-a soft metal-becomes 10 \\ times stronger than high-grade steel.
}

\section{by Arianna E. Gleason*}

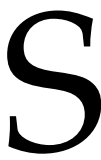
trength-the maximum stress a material can withstand before it fails or deforms-is a fundamental property of a material. Strength is typically measured under static conditions, but it can change significantly when a large stress is applied rapidly. Understanding this dynamic strength behavior would benefit applications ranging from the design of armory and bullet-proof vests to the development of fusion schemes based on the laserdriven compression of a fuel pellet. Now, Andrew Krygier of Lawrence Livermore National Laboratory in California and colleagues have performed dynamic strength measurements on lead $(\mathrm{Pb})$ and on a series of $\mathrm{Pb}$-based alloys at some of the highest pressures ever explored, about $400 \mathrm{GPa}$. Exploiting the powerful lasers available at the National Ignition

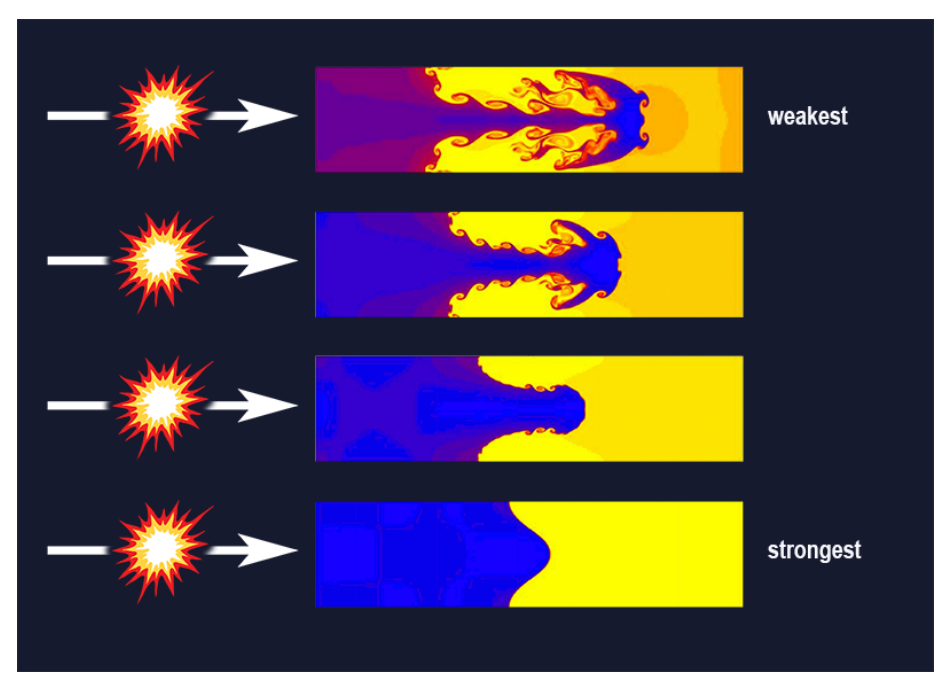

Figure 1: The application of stress to a solid causes the material to flow-a phenomenon known as Rayleigh-Taylor instability. This flow causes ripples patterned on the material surface to grow, forming jet-like structures coming off the surface. (Shengtai Li and Hui Li/Los Alamos National Laboratory; adapted by APS/Joan Tycko)

*Department of Geological Sciences, Stanford University, Stanford, CA, USA
Facility (NIF), the team compressed the samples and characterized their strength by tracking the growth of tiny ripples of material on the sample surface [1]. They found that the large pressure and rate of deformation, or strain, produce a remarkable "hardening" of $\mathrm{Pb}$ : this normally soft metal became 250 times stronger when compressed. This hardening is due to a mechanism that might be useful for tuning the properties of important industrial materials, like steel.

How strong is a material? There are different "flavors" of strength: shear, tensile, compressive, or yield strength-each of which has certain values at ambient conditions. But strength can change dramatically under extreme conditions of pressure and temperature. And strength under static conditions (with stress exerted over long periods of time-from days to decades) may be different from dynamic strength (measured when the loading process is shorter than a second). To improve material performance in a broad range of applications, researchers would like to better understand the microscopic mechanisms behind these differences.

Knowing the strength of a material at extreme conditions or varying strain rates is challenging. There are empirical functional formulas to extrapolate the values under extreme conditions from those at ambient conditions, but they are often unreliable. Researchers have thus developed a number of techniques-such as x-ray imaging, diffraction, and laser interferometry - to characterize strength-related properties. In particular, interferometric probes can be coupled with dynamic compression platforms (like gas guns and laser-driven shocks) to observe stress-induced shear waves, whose speed can be related to the strength of the material $[2,3]$. These dynamic measurements, however, remain difficult, in particular for soft metals like $\mathrm{Pb}$, which typically have a low melting temperature: When compressed, the unavoidable heating can cause the material to melt, like the soft solder used in electronics and plumbing.

Krygier and his colleagues used a complex and clever setup to address the challenge of bringing a soft material like $\mathrm{Pb}$ to extremely high pressures and strain rates without melting it. They pointed the 160 laser beams of the NIF at a sample roughly the size of a small blueberry. By tailoring the temporal shape of the laser pulses-an approach called ramp compression-they induced a gradual increase in sample pressure over tens of nanoseconds, which allowed the temperature to stay below the melting point of $\mathrm{Pb}$. They then characterized the samples through a stan- 


\section{Physīcs}

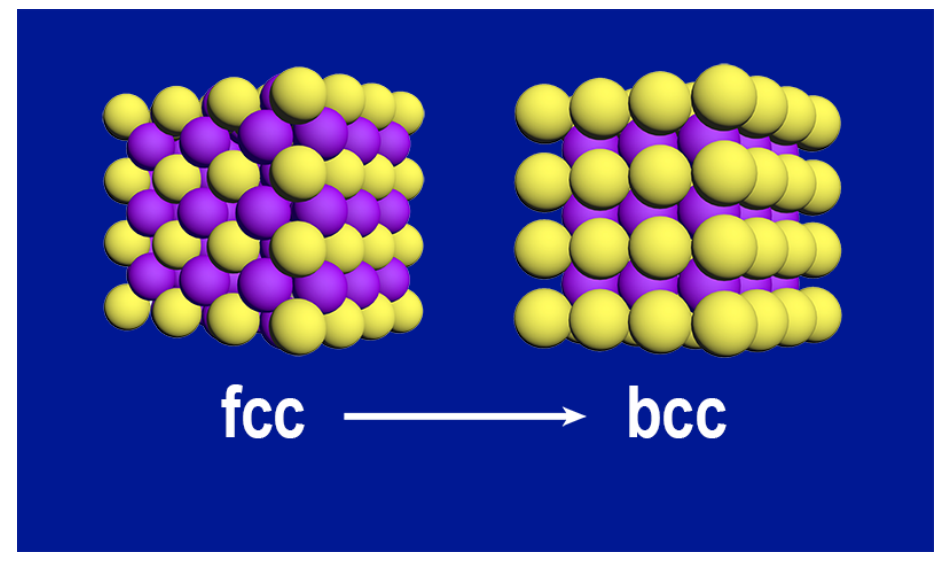

Figure 2: Calculations based on a dynamic stress model suggest that the observed hardening is due a transition from a face-centered cubic (fcc) to a body-centered cubic (bcc) crystalline structure. (APS/Joan Tycko)

dard approach based on using $x$ rays to observe the growth of ripples that were patterned on the sample surface [4-6]. As the wave of compression transited the sample, it caused the ripples to grow through hydrodynamical instabilities, known as Rayleigh-Taylor instabilities, forming jets that carried material off of the surface (Fig. 1). Crucially, the lengths and shapes of these ripples depend on the sample strength, viscosity, and on the flow of material as it deforms. The researchers tracked the ripple evolution by taking radiographic snapshots of the samples at delays of several tens of nanoseconds with respect to the compressing laser pulses.

The x-ray images reveal a slow ripple growth, which suggests that $\mathrm{Pb}$ got much stronger under the experimental conditions. How could this be, since $\mathrm{Pb}$ is very soft at room conditions? To derive a quantitative strength characterization and provide microscopic insights into the observed behavior, the authors applied an available dynamic stress model, called the improved Steinberg-Guinan (ISG) model, which can account for changes in the sample's crystalline structure. The simulations suggest the following (Fig. 2): At ambient conditions, $\mathrm{Pb}$ 's lattice has a face-centered cubic (fcc) structure, which allows dislocations (or imperfections) in the lattice to easily glide through the crystal, making it easily malleable. $\mathrm{As} \mathrm{Pb}$ is compressed, the $\mathrm{Pb}$ structure goes through a series of changes, finally reaching a body-centered cubic structure (bcc). A bcc structure-like that found in hard metals like tantalum-does not allow dislocations to glide like in fcc crystal. Instead, the bcc lattice responds to an applied stress by twinning-forming intergrown crystals that are symmetric images of one another [7,8]. Fitting the data with the ISG model, Krygier and co-workers estimate that compressed $\mathrm{Pb}$ attains a Hulk-like strength of nearly 4 $\mathrm{GPa}-250$ times larger than $\mathrm{Pb}$ at ambient conditions and about 10 times larger than any known high-strength steel.
The researchers also investigated a series of $\mathrm{Pb}$ alloys, in which $\mathrm{Pb}$ is combined with antimony to increase strength for applications like lead-acid batteries and bullets. At ambient conditions, alloying can increase strength by a factor of 4 . Would a similar gain hold in the dynamic, highpressure regime? Surprisingly, the authors found that all alloys reached the same strength as $\mathrm{Pb}$ when compressed. In other words, alloying does not provide the same hardening advantages in the dynamic regime as it does under static conditions.

The dramatic strength change observed by the authors points at a new dynamic hardening paradigm, whereby weak materials become stronger through a structural change of their lattice. The results showcase NIF's potential to characterize the strength of materials-even soft ones with low melting temperatures-at extreme pressures, paving the way for the investigation of a broad class of solids. The new experimental platform will provide a much-needed tool to validate models and improve our ability to design materials whose properties are tuned to optimize their performance in a given application.

The fcc-to-bcc change of $\mathrm{Pb}$ was inferred indirectly by fitting a model to the data, but it would be more informative to observe it directly with appropriate probes. This observation could be done with ultrafast $\mathrm{x}$-ray diffraction (XRD) - which can probe structural changes in the lattice and could thus deliver direct evidence of the fcc-to-bcc transformation. Time-resolved x-ray imaging could also probe nano-to-mesoscale changes in the solid's microstructure. Opportunities to perform these experiments with unprecedented spatial and temporal resolution are offered by $x$-ray free-electron lasers (XFELs) - sources with unmatched coherence and brilliance, delivering pulses shorter than $100 \mathrm{fs}$. By borrowing the setup design of certain static compression schemes [9], in which $x$ rays probe the direction along which a compressed material is weakest, XFEL experiments may be able to pinpoint the exact deformation mechanisms and characterize dynamic strength and even strength anisotropy (lattice direction-dependent strength).

This research is published in Physical Review Letters.

\section{REFERENCES}

[1] A. Krygier et al., "Extreme hardening of $\mathrm{Pb}$ at high pressure and strain rate," Phys. Rev. Lett. 123, 205701 (2019).

[2] A. E. Gleason et al., "Lithographically fabricated gratings for the interferometric measurement of material shear moduli under extreme conditions," J. Vac. Sci. Technol. B 30, 06F306 (2012).

[3] T. Jiao and R. J. Clifton, "Measurement of the response of an elastomer at pressures up to $9 \mathrm{GPa}$ and shear-rates of $10^{5}-$ $10^{6} \mathrm{~s}^{-1}$," J. Phys.: Conf. Proc. 500, 112036 (2014).

[4] S. Zybin et al., "Molecular dynamics simulations of the Richtmyer-Meshkov instability in shock loaded solids," in Shock compression of Condensed Matter-2005, AIP Conf. Proc. No. 
845, edited by M. Furnish, M. Elert, T. Russell, and C. White (AIP, Melville, 2006), p. 437.

[5] S. M. Pollaine, B. A. Remington, H. S.Park, S. T. Prisbrey, and R. M. Cavallo, "Designfor solid-state Rayleigh-Taylor experiments in tantalum at Omega," J. Phys.: Conf. Ser. 244, 042016 (2010).

[6] B. A. Remington et al., "Rayleigh-Taylor instabilities in highenergy density settings on the National Ignition Facility," Proc. Natl. Acad. Sci. U.S.A. 116, 18233 (2018).

[7] C. E. Wehrenberg et al., "In situ x-ray diffraction measurement of shock-wave-driven twinning and lattice dynamics," Nature 550,
496 (2017).

[8] J. Chen, E. N. Hahn, A. M. Dongare, and S. J. Fensin, "Understanding and predicting damage and failure at grain boundaries in BCC Ta," J. Appl. Phys. 126, 165902 (2019).

[9] A. E. Gleason and W. L. Mao, "Strength of iron at core pressures and evidence for a weak Earth's inner core," Nat. Geo. 6, 571 (2013).

10.1103/Physics.12.125 